\title{
New genetic insights into the development of alcohol-related cirrhosis
}

Results from a genome-wide association study (GWAS) report variants in two previously unrelated genes, TM6SF2 and $M B O A T 7$, and confirm another one in PNPLA3 as important risk loci for alcohol-related cirrhosis.

Although hepatic steatosis occurs in most people with a history of chronic alcohol misuse, only 10-15\% develop cirrhosis in the long term. To assess in more detail why outcomes vary substantially despite similar drinking habits, investigators performed a twostage GWAS, which compared patients with alcohol-related cirrhosis and heavy drinkers without cirrhosis (controls). The first stage was conducted separately in Germany and the UK (712 patients, 1,426 controls); the second stage validated results in a meta-analysis of independent samples.

The study confirmed a strong association for a genetic variant in the gene PNPLA3, which has previously been reported as a predictor of hepatic fat content and disease progression in NAFLD. Two novel risk loci in TM6SF2 and MBOAT7 were also identified.

"With the data from our study, the genetic background of alcoholic cirrhosis has become clearer and seems to be confined to intrahepatic fat turnover, as all genetic variants found in our study relate to fat metabolism and associated inflammatory processes," explains corresponding author Felix Stickel.

In addition, the results suggest that pathogenic mechanisms might be shared between NAFLD and alcoholrelated cirrhosis, and similar therapeutic approaches might be beneficial.

Christine Weber

Original article Buch, S. et al. A genome-wide association study confirms PNPLA3 and identifies TM6SF2 and MBOAT7 as risk loci for alcohol-related cirrhosis. Nat. Genet. doi:10.1038/ng.3417 